\title{
Central neurogenic hyperventilation
}

回

Figure Extensive pontine lesions in CLIPPERS resulting in central neurogenic hyperventilation
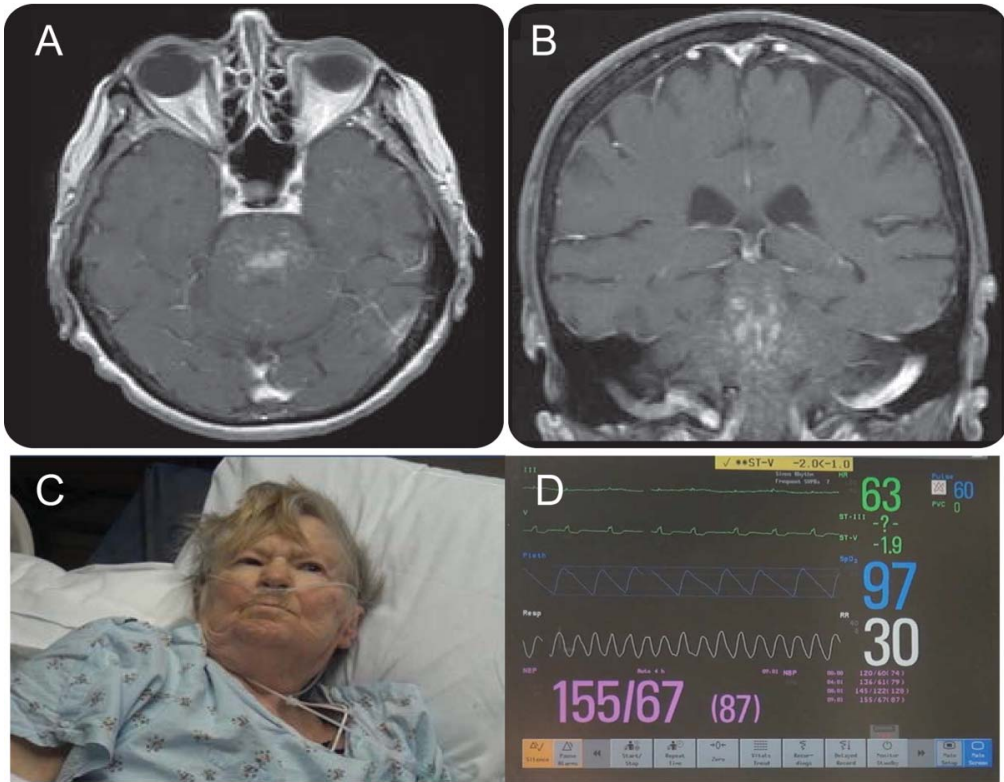

Axial (A) and coronal (B) T1 postgadolinium brain MRI shows characteristic punctate and curvilinear enhancement peppering the brainstem in chronic lymphocytic inflammation with pontine perivascular enhancement responsive to steroids syndrome (CLIPPERS). Presumed medial pontine nuclear involvement resulted in central neurogenic hyperventilation (D) with inability to breath-hold (C).

A 79-year-old woman developed subacute progressive gait instability. Brain MRI demonstrated patchy nodular enhancement within the brainstem and cerebellum (figure). Brain biopsy was consistent with chronic lymphocytic inflammation with pontine perivascular enhancement responsive to steroids syndrome (CLIPPERS). ${ }^{1}$ Questioning revealed shortness of breath (figure, video). Blood gas on room air revealed $\mathrm{pH} 7.59, \mathrm{PaCO}_{2} 16$, $\mathrm{PaO}_{2} 103$, and $\mathrm{HCO}_{3} 15$.

Central neurogenic hyperventilation, often associated with brainstem lymphoma, rare in noncomatose patients, and never described in CLIPPERS, results from released respiratory inhibition from medial pontine nuclear damage. ${ }^{2}$ Being comfortably tachypneic can be due to a pontine lesion.

Christopher L. Kramer, MD, Eelco F.M. Wijdicks, MD, PhD

From Mayo Clinic, Rochester, MN.

Author contributions: Dr. Kramer: drafted and revised manuscript. Dr. Wijdicks: design and conceptualization, revised manuscript. Study funding: No targeted funding reported.

Supplemental data at Neurology.org
Disclosure: The authors report no disclosures relevant to the manuscript. Go to Neurology.org for full disclosures.

Correspondence to Dr. Kramer: kramer.christopher1@mayo.edu

1. Pittock S, Debruyne J, Krecke KN, et al. Chronic lymphocytic inflammation with pontine perivascular enhancement responsive to steroids (CLIPPERS). Brain 2010;133:2626-2634.

2. Plum F, Swanson AO. Central neurogenic hyperventilation in man. AMA Arch Neurol Psychiatry 1959;81:535-549. 


\title{
Neurology
}

\author{
Central neurogenic hyperventilation \\ Christopher L. Kramer and Eelco F.M. Wijdicks \\ Neurology 2014;83;376 \\ DOI 10.1212/WNL.0000000000000624
}

This information is current as of July 21, 2014

$\begin{array}{ll}\begin{array}{l}\text { Updated Information \& } \\ \text { Services }\end{array} & \begin{array}{l}\text { including high resolution figures, can be found at: } \\ \text { http://n.neurology.org/content/83/4/376.full }\end{array} \\ \text { Supplementary Material } & \text { Supplementary material can be found at: } \\ \text { http://n.neurology.org/content/suppl/2014/07/20/83.4.376.DC1 } & \\ & \text { This article cites } 2 \text { articles, } 0 \text { of which you can access for free at: } \\ \text { heftp://n.neurology.org/content/83/4/376.full\#ref-list-1 } & \\ & \text { This article, along with others on similar topics, appears in the } \\ \text { following collection(s): } & \text { All Immunology } \\ \text { http://n.neurology.org/cgi/collection/all_immunology } & \text { Clinical neurology examination } \\ & \text { http://n.neurology.org/cgi/collection/clinical_neurology_examination } \\ & \text { Critical care } \\ & \text { http://n.neurology.org/cgi/collection/critical_care } \\ \text { MRI } & \text { http://n.neurology.org/cgi/collection/mri } \\ & \text { Information about reproducing this article in parts (figures,tables) or in } \\ & \text { its entirety can be found online at: } \\ & \text { http://www.neurology.org/about/about_the_journal\#permissions } \\ & \text { Information about ordering reprints can be found online: } \\ \text { http://n.neurology.org/subscribers/advertise }\end{array}$

Neurology ${ }^{\circledR}$ is the official journal of the American Academy of Neurology. Published continuously since 1951, it is now a weekly with 48 issues per year. Copyright () 2014 American Academy of Neurology. All rights reserved. Print ISSN: 0028-3878. Online ISSN: 1526-632X.

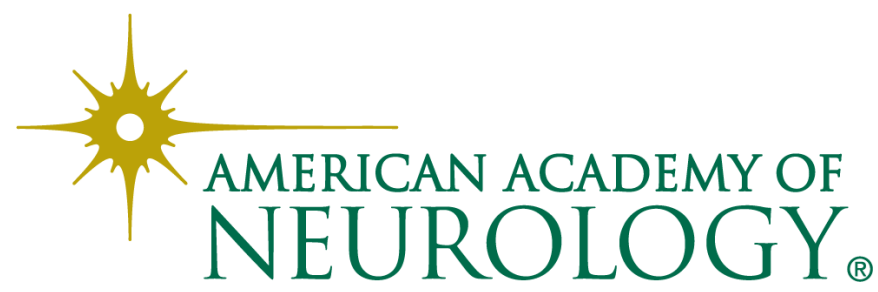

